\title{
Diferenciação morfométrica entre larvas de Amblyomma sculptum Berlese, 1888 e Amblyomma dubitatum Neumann, 1899
}

\author{
[Morphometric differentiation between larvae of Amblyomma sculptum Berlese, \\ 1888 and Amblyomma dubitatum Neumann, 1899] \\ J. Brites-Neto ${ }^{1,2}$, J. Brasil ${ }^{2}$, G.A.C.G. Takeda ${ }^{3}$, A.C. Guillen ${ }^{4}$, \\ M.B. Labruna ${ }^{5}$, A. Pinter ${ }^{3}$ \\ ${ }^{1}$ Escola Superior de Agricultura Luiz de Queiroz/USP - Piracicaba, SP \\ ${ }^{2}$ Secretaria de Saúde - Americana, SP \\ ${ }^{3}$ Superintendência de Controle de Endemias - São Paulo, SP \\ ${ }^{4}$ Universidade Federal do Paraná - Curitiba, PR \\ ${ }^{5}$ Faculdade de Medicina Veterinária e Zootecnia/USP - São Paulo, SP
}

\begin{abstract}
RESUMO
Os carrapatos estão envolvidos em processos biológicos de uma grande variedade de organismos patogênicos. O gênero Amblyomma é o de maior importância médica, com a espécie Amblyomma sculptum Berlese, 1888 envolvida no ciclo de transmissão da febre maculosa brasileira (FMB). Neste estudo, objetivou-se a validação molecular para uma diferenciação na característica morfométrica e no tamanho de idiossoma de larvas de duas espécies de carrapatos, Amblyomma dubitatum Neumann, 1899 e A. sculptum. Larvas não alimentadas foram coletadas em duas áreas de transmissão para FMB, por meio da técnica de armadilha atrativa de $\mathrm{CO}_{2}$. Foram identificadas em nível de espécie por morfometria comparativa, análise molecular por PCR e sequenciamento genômico, com validação pela análise de concordância pelo teste Kappa. As larvas de A. dubitatum apresentaram um comprimento significativamente maior que as larvas de A. sculptum. Embora nenhuma outra espécie do gênero Amblyomma tenha sido testada neste estudo, essa técnica poderá ser utilizada nos locais onde levantamentos acarológicos prévios, baseados nos estádios de ninfa e adultos, indicaram a presença de apenas A. sculptum e A. dubitatum, geralmente mantidos por capivaras. Digno de nota, essa condição é muito comum ao longo das áreas endêmicas para FMB na região Sudeste do Brasil.
\end{abstract}

Palavras-chave: carrapatos, idiossoma, taxonomia, sequenciamento genético, capivaras, febre maculosa

\begin{abstract}
Ticks are involved in biological processes of a wide variety of pathogenic organisms. The genus Amblyomma presents the greatest medical importance, with the species Amblyomma sculptum Berlese, 1888 involved in the transmission cycle of Brazilian Spotted Fever (BSF). In this study, we performed a molecular validation of the morphometric differentiation based on the idiosomal length of the larvae of $A$. dubitatum and A. sculptum. Unfed larvae were collected in two BSF-transmission areas, using the attractive $\mathrm{CO}_{2}$ trap technique. Larvae were identified at the species level by comparative morphometry, molecular analysis by PCR and genomic sequencing, with validation through agreement analysis by the Kappa test. The larvae of A. dubitatum showed a significantly longer idiosomal length than A. sculptum larvae. Although no other species of the genus Amblyomma has been tested in this study, this technique can be applied to places where previous acarological surveillances based on adult and nymphal ticks stages have indicated the presence of only A. sculptum and A. dubitatum, usually sustained by capybaras. Noteworthy, this condition is very common among many BSF-endemic areas in southeastern Brazil.
\end{abstract}

Keywords: ticks, idiosome, taxonomy, genetic sequencing, capybaras, spotted fever

Recebido em 31 de janeiro de 2017

Aceito em 5 de julho de 2017

E-mail: samevet@yahoo.com.br 


\section{INTRODUÇÃO}

Os carrapatos estão envolvidos em processos biológicos na transmissão de grande variedade de organismos patogênicos, sendo superiores a qualquer outro grupo de artrópodes, embora os mosquitos possam transmitir patógenos que infectam um maior número de pessoas e causem doenças com maior potencial de gravidade em animais e seres humanos (Sonenshine e Roe, 2014). Dentre essas doenças, as zoonoses são as principais causas de morbidade e mortalidade no mundo, tendo os carrapatos como vetores mais ativos do que qualquer outro grupo de invertebrados (Pfäffle et al., 2013).

Carrapatos ixodídeos estão distribuídos em todo o mundo, com espécies do gênero Amblyomma Kock, 1844, observadas em muitos casos de parasitismo humano (Guglielmone et al., 2014). No Brasil, o gênero Amblyomma é o de maior importância médica, com 32 espécies endêmicas distribuídas nas diversas regiões do país (DantasTorres et al., 2009; Krawczak et al., 2015; Martins et al., 2016). Duas dessas espécies, Amblyomma sculptum Berlese, 1888 e A. aureolatum (Pallas, 1772), estão envolvidas como importantes vetores, no ciclo de transmissão da febre maculosa brasileira (FMB), uma antropozoonose de notificação compulsória, causada pela bactéria Rickettsia rickettsii, que apresenta características epidemiológicas de elevada endemicidade e alta letalidade, em decorrência de fatores relacionados ao tratamento tardio, ao uso de antibióticos menos eficazes e à incidência de cepas mais virulentas de $R$. rickettsii na população humana do estado de São Paulo (Ogrzewalska et al., 2012; Labruna et al., 2014).

Essa zoonose é caracterizada por uma doença infecciosa aguda, de gravidade variável, apresentando um período de incubação de dois14 dias, e sinais clínicos evidenciados por febre contínua, calafrios, prostração, mal-estar, mialgia, artralgia, cefaleia, erupções cutâneas maculopapulares e distúrbios hemostáticos (Pinter et al., 2011), com uma taxa de letalidade variando entre 20 e $40 \%$, a qual, nos casos não tratados, pode chegar a 80\% (Parola et al., 2013; Labruna, 2013).

Em relação ao seu principal vetor, estudos recentes reavaliaram o status taxonômico da espécie A. cajennense por meio de análises morfológicas e moleculares, estabelecendo um complexo de seis espécies: A. cajennense sensu stricto, Amblyomma tonelliae Nava et al., 2014, Amblyomma interandinum Beati et al., 2014, Amblyomma patinoi Labruna., 2014, Amblyomma mixtum Koch, 1844 e A. sculptum (Beati et al., 2013; Estrada-Peña et al., 2014; Nava et al., 2014). Dentro desse complexo, foram validadas para o Brasil as espécies $A$. cajennense sensu stricto e A. sculptum, e para o estado de São Paulo, somente a espécie $A$. sculptum. Por conseguinte, a espécie A. sculptum tem a capivara (Hydrochoerus hydrochaeris Linnaeus, 1776) como um hospedeiro primário para todos os seus estádios parasitários (Krawczak et al., 2014), desempenhando um importante papel na epidemiologia da doença como um competente amplificador da circulação do agente etiológico no ambiente (Souza et al., 2009).

Outra espécie desse gênero, A. dubitatum, apresenta um papel indeterminado como vetor da FMB e foi associada com uma rickéttsia de patogenicidade ainda não evidenciada para humanos, Rickettsia sp. strain Pampulha (Almeida et al., 2011), e outra não patogênica e não pertencente ao grupo da febre maculosa, $R$. bellii (Sakai et al., 2014). Não obstante, $A$. dubitatum também pode eventualmente parasitar pessoas, visto que Labruna et al. (2007) observaram infestação por todos os estádios dessa espécie em humanos. Capivaras infestadas por A. sculptum encontram-se frequentemente coinfestadas por A. dubitatum, que também tem esse roedor como hospedeiro primário (Nava et al., 2010; Labruna, 2013), o que suscita maior investigação quanto à sua relação com $A$. sculptum e a bactéria $R$. rickettsii (Szabó et al., 2013; Matias et al., 2015).

As pesquisas acarológicas realizadas para levantamento de espécies de ixodídeos nas regiões endêmicas para FMB do estado de São Paulo necessitam de referências técnicas eficientes e práticas para identificação taxonômica de todos os estádios de vida de carrapatos prevalentes em áreas de transmissão. Para as formas adultas de espécies de Amblyomma, existem as chaves dicotômicas traduzidas e modificadas por Onofrio et al. (2006), e para as formas imaturas, há a chave de identificação para ninfas de Martins et al. (2010). 
No entanto, há poucas espécies descritas no estádio larval, em que os caracteres morfológicos são pouco informativos e as descrições baseadas em quetotaxia não são suficientes para separação das espécies. Apesar de avanços terem sido promissores por meio de chaves de identificação para larvas com base em porotaxia, no que diz respeito à forma, o número e a topografia dos poros, os procedimentos atuais se pautam em técnicas em que os estádios imaturos de carrapatos recolhidos naturalmente são criados em animais de laboratório até a fase adulta, para sua correta identificação, uma vez que as chaves de identificação para larvas são incompletas (Barbieri et al., 2006; Pallini et al., 2007).

Considerando essas limitações, na presente análise, objetivou-se a validação molecular para uma diferenciação na característica morfométrica e no tamanho de idiossoma de larvas de duas espécies de carrapatos, A. dubitatum e $A$. sculptum, nas áreas com prevalência de adultos dessas espécies, em regiões de transmissão para FMB.

\section{MATERIAL E MÉTODOS}

Larvas de carrapatos do gênero Amblyomma foram coletadas mediante autorização para atividades com finalidade científica, SISBIO $n^{\circ}$
20562, em duas áreas de transmissão para FMB, situadas no estado de São Paulo. Essas áreas são caracterizadas por matas ciliares do Rio Jaguari e do Rio Piracicaba e estão situadas na localidade do Sobrado Velho (22 41'16"S e 47 15'09'WO; $516 \mathrm{~m})$ e Carioba $\left(22^{\circ} 41^{\prime} 75^{\prime}\right.$ 'S e $47^{\circ} 19^{\prime} 30^{\prime \prime}$ WO; $496 \mathrm{~m})$, respectivamente, em zona rural e periurbana do município de Americana.

Foram realizadas pesquisas acarológicas por meio da técnica de armadilha atrativa de $\mathrm{CO}_{2}$ (Wilson et al., 1972; Ramos et al., 2014). A utilização das armadilhas de $\mathrm{CO}_{2}$ é sensível e eficiente para a coleta de todos os estádios de $A$. sculptum e A. dubitatum (Souza et al., 2006).

No período de 04/08/2011 a 06/08/2013, foram distribuídas 88 armadilhas de $\mathrm{CO}_{2}$ em vários pontos nas duas áreas, com tempo médio de permanência no solo de $1 \mathrm{~h} 30$.

As larvas coletadas foram identificadas em nível de espécie, em estereomicroscópio binocular com aumento de 30x, com base no tamanho do corpo de cada indivíduo. Para isso, foi utilizada a identificação morfométrica comparativa, considerando que as larvas de $A$. dubitatum são notavelmente maiores em relação às larvas de $A$. sculptum (Brites-Neto et al., 2013) (Fig. 1).

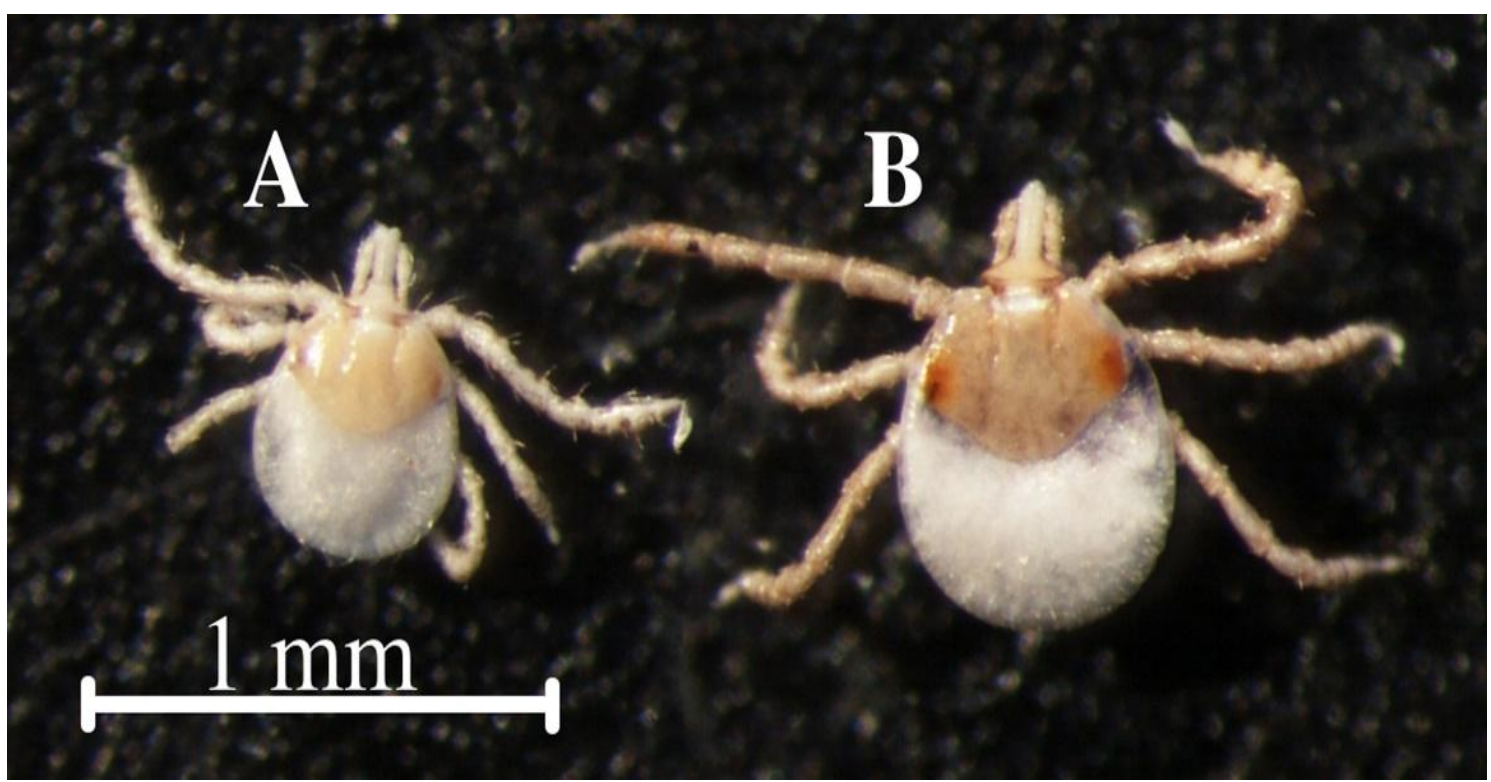

Figura 1. Visualização da diferença morfométrica entre os espécimes larvas de $A$. sculptum (A) e $A$. dubitatum (B). 
As larvas foram separadas em dois grupos. O primeiro continha larvas identificadas como $A$. sculptum, enquanto o segundo continha larvas identificadas como A. dubitatum.

De cada um dos dois grupos, 30 larvas foram aleatoriamente selecionadas e transferidas individualmente para microtubos de $0,5 \mathrm{~mL}$ (Axygen, Life Science Brand, EUA) contendo álcool isopropílico.

As larvas foram retiradas do álcool isopropílico, deixadas para secar em papel-toalha pelo período de 10 minutos e transferidas individualmente para lamínulas de vidro descartáveis. As lamínulas foram examinadas em estereomicroscópio com câmera acoplada e conectado a um computador com software de análise de imagem (NIS-Elements F, Nikon, Japão). A medição individual do comprimento longitudinal do idiossoma foi realizada com as larvas posicionadas em decúbito ventral.

As larvas foram transferidas individualmente para novos microtubos de $0,5 \mathrm{~mL}$ previamente preenchidos com 50 microlitros de tampão Tris$\mathrm{HCl} 10 \mathrm{mM}$, pH 7,4 e EDTA 1mM, pH 8,0 (TE). As larvas foram maceradas com micropistilo plástico descartável e todos os tubos foram transferidos ao termociclador (Mastercycler Gradient ${ }^{\circledR}$ Eppendorf $\left(\right.$ ) ) e expostos a $99^{\circ} \mathrm{C}$ pelo período de 15 minutos. Nessa etapa, ocorreu uma desnaturação e inativação de enzimas e outras proteínas que apresentam potencial de inibição na reação de PCR e de rompimento de células e exposição do DNA genômico e mitocondrial.

A PCR foi conduzida utilizando-se o kit comercial (Master Mix Qiagen(C)). A quantidade de reagentes utilizados por microtubo seguiu o protocolo do fabricante, que consistiu de $12,5 \mu \mathrm{L}$ de Master mix; $0,5 \mu \mathrm{L}$ do primer forward a $10 \mathrm{nM} ; 0,5 \mu \mathrm{L}$ do primer reverse a $10 \mathrm{nM} ; 9,5 \mu 1$ de água fornecida no kit comercial e $2 \mu \mathrm{L}$ de amostra, compondo um volume total de $25 \mu \mathrm{L}$. Para o controle negativo da PCR, foi utilizada amostra de água destilada, e para o controle positivo, foi utilizado material genético de $A$. aureolatum previamente testado.

Utilizou-se a metodologia de amplificação e o sequenciamento do fragmento do gene ribossômico-mitocondrial $16 \mathrm{~S}$ rRNA. O DNA extraído de cada larva de carrapato foi individualmente submetido à reação de PCR. Os oligonucleotídeos iniciadores específicos para amplificação de um fragmento de 460 pares de base foram, FORWARD: 5'-CTGCTCAATGATTTTTTAAATTGCTGTGG-3' (16S +1, Black e Piesman, 1994) e REVERSE: 5'-CCGGTCTGAACTCAGATCAAGT-3' (16S -1, Black e Piesman, 1994), como descrito por Mangold et al. (1998).

A reação em cadeia foi conduzida em termociclador e o programa de amplificação foi utilizado como sugerido por Mangold et al. (1998), adaptado às exigências do fabricante dos reagentes Master mix, adicionando-se uma etapa de 15 minutos a $95^{\circ} \mathrm{C}$ ao protocolo original.

Ao final da reação, foram adicionados $2 \mu \mathrm{L}$ do tampão carregador "blue loading dye", em uma alíquota de $8 \mu \mathrm{L}$ de cada amostra. $\mathrm{O}$ volume final de $10 \mu \mathrm{L}$ foi aplicado individualmente em poços com gel de agarose $1,5 \%$, previamente preparado com solução de Tris/ácido bórico/EDTA (TBE) e tratado com o revelador GelRed ${ }^{\circledR}$.

Para a comparação com o padrão de massa, utilizou-se o marcador de massa molecular DNA Ladder Invitrogen $®$, aplicado sempre na primeira coluna de cada gel. Em seguida, o gel foi submetido à eletroforese pelo intervalo de $40 \mathrm{~min}$, sob corrente elétrica de 0,05 A e tensão de $6 \mathrm{v} / \mathrm{cm}$.

Após o término da eletroforese, o gel foi submetido à incidência de luz ultravioleta (UV) em transiluminador DyNA Light Labnet ${ }^{\circledR}$, com o objetivo de revelar fragmentos de DNA amplificados, assim como o marcador molecular utilizado.

As amostras amplificadas foram purificadas com reagente EXOSAP, seguindo protocolo do fabricante, e encaminhadas para sequenciamento no Centro de Estudos do Genoma Humano da USP, onde a reação utilizada foi a do método didesoxi terminal, de acordo com Sanger et al. (1977), com reagentes do Kit BigDye ABI PrismTM Dye Terminator Cycle Sequencing Reading Reaction - Applied Biosystems.

Com os dados do sequenciamento genômico, os cromatogramas senso e antissenso gerados foram alinhados por amostra, utilizando-se o programa de computador Geneious 10.0.2 Biomatters Ltd. 
As sequências consenso foram alinhadas e comparadas ao banco de dados do GenBank no National Center for Biotechnology Information (NBCI), usando o programa Basic Local Alignment Search Tool (BLAST).

Os dados da medição longitudinal das larvas foram comparados pelo teste de Mann-Whitney U, e a concordância do resultado do sequenciamento com o resultado da microscopia óptica foi comparada pelo teste Kappa, sendo os testes estatísticos executados no programa SOFA 1.4.3 Paton-Simpson \& Associates Ltd.

\section{RESULTADOS}

Durante o processo de coleta por armadilhas de $\mathrm{CO}_{2}$, foram capturadas 4.347 larvas de carrapatos. Seguindo a identificação morfométrica comparativa, 3.812 larvas foram identificadas como A. sculptum, e 535 larvas como A. dubitatum.

As larvas identificadas previamente em estereomicroscópio óptico como pertencentes à espécie $A$. dubitatum apresentaram um comprimento médio do idiossoma de $0,609 \mathrm{~mm}$ $(0,554 \mathrm{~mm}$ - 0,666mm), com um valor de mediana de $0,607 \mathrm{~mm}$ e um desvio-padrão de $\pm 0,03 \mathrm{~mm}$. As larvas identificadas previamente como pertencentes à espécie A. sculptum apresentaram um comprimento médio do idiossoma de 0,469mm $(0,42 \mathrm{~mm}$ - 0,498mm), com um valor de mediana de $0,474 \mathrm{~mm}$ e um desvio-padrão de $\pm 0,018 \mathrm{~mm}$ (Tab. 1).

Tabela 1. Medidas do idiossoma de larvas identificadas como A. dubitatum e A. sculptum

\begin{tabular}{ccccc}
\hline Grupo & Média $(\mathrm{mm})^{*}$ & Mediana $(\mathrm{mm})$ & Max. $(\mathrm{mm})$ & Min. $(\mathrm{mm})$ \\
\hline A. dubitatum & $0,609^{\mathrm{a}}$ & 0,607 & 0,666 & 0,554 \\
A. sculptum & $0,469^{\mathrm{b}}$ & 0,474 & 0,498 & 0,42 \\
\hline
\end{tabular}

*Letras diferentes representam diferença estatística avaliada no teste de Mann-Whitney U, valor de $\mathrm{P}<0,001$ e U = 0 .

Houve diferença significativa entre as medidas do tamanho do idiossoma dos grupos, e o grupo de larvas identificadas como $A$. dubitatum apresentou-se com um comprimento de idiossoma maior $(\mathrm{P}<0,001 ; \mathrm{U}=0)$ do que o grupo identificado como A. sculptum (Fig. 2).

O sequenciamento de parte do gene mitocondrial 16S rRNA de cada uma das 60 larvas foi avaliado primeiramente de forma individual, sendo retirada manualmente a sequência referente aos primers e selecionada parte do sequenciamento que obteve alta qualidade na definição das bases no cromatrograma. Posteriormente, foi feito um agrupamento entre as sequências em que houve um alinhamento local de $100 \%$.

Todas as 30 sequências consenso das larvas previamente identificadas como A. dubitatum alinharam-se entre si com $100 \%$ de similaridade, formando uma sequência consenso de 301 pares de base. Essa sequência apresentou alta similaridade com sequências de A. dubitatum disponíveis no GenBank, sendo $100 \%$ com o registro GU301912.1 e 99,6\% com o registro KU894374.1.

Todas as 30 sequências consenso das larvas previamente identificadas como A. sculptum alinharam-se entre si com $100 \%$ de similaridade, formando uma sequência consenso de 359 pares de base. Essa sequência apresentou $100 \%$ de similaridade com sequências de A. sculptum disponíveis no GenBank, sendo $100 \%$ com os registros KT820361.1 e FJ424404.1. Vale ressaltar que o registro FJ424404.1 foi inicialmente descrito como A. cajennense, sendo anterior ao artigo de Beati et al. (2013), tratandose na verdade de A. sculptum.

Os dados do sequenciamento genético concordam, portanto, com os resultados dos dados de medição do idiossoma das larvas, assim como da identificação morfológica prévia, tendo apresentado, dessa forma, um valor de concordância Kappa geral igual a 1,0 com valor de $\mathrm{P}<0,001$ e um intervalo de $95 \%$ de confiança de $(0,747 ; 1,0)$. 


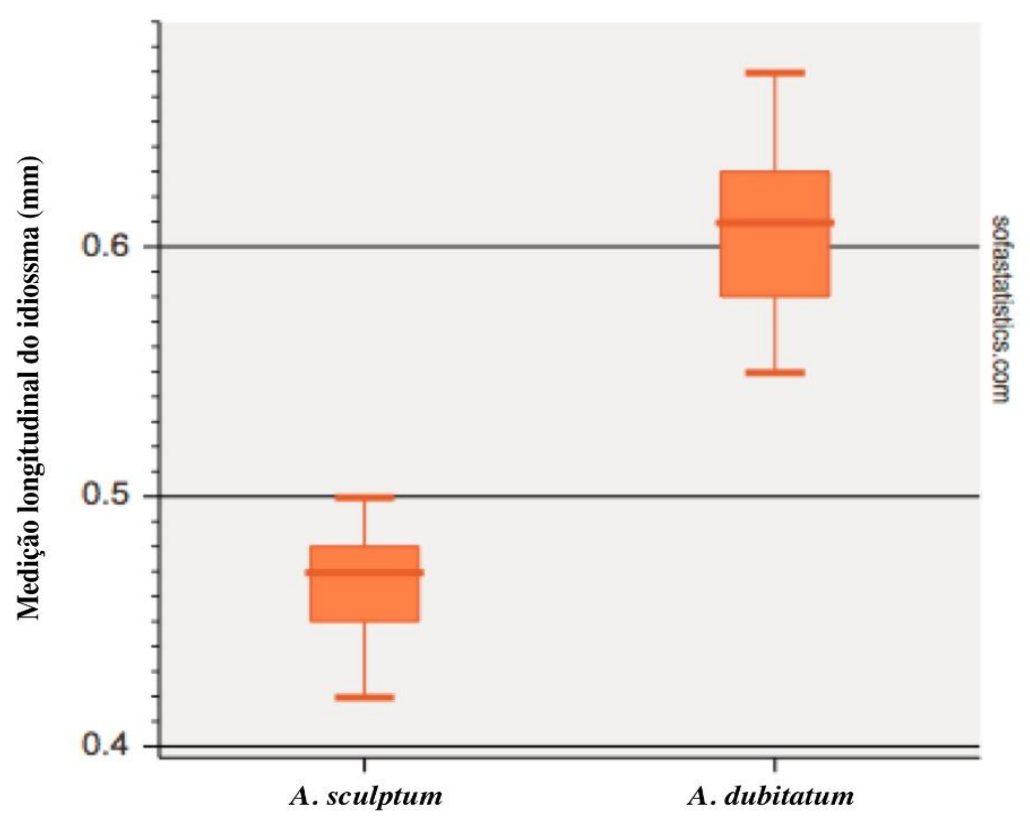

Figura 2. Comprimento do idiossoma de A. sculptum e A. dubitatum.

\section{DISCUSSÃO}

A correta identificação da espécie de carrapato existente em um determinado local provável de infecção (LPI) é de suma importância e pode alterar significativamente o risco atribuído a esse local quanto à ocorrência de FMB (Pinter et al., 2016).

Este estudo demonstrou que é possível diferenciar, com alto grau de validação, as espécies A. sculptum e A. dubitatum nas fases larvais, visto que a seleção dos espécimes pelo tamanho do idiossoma conferiu acerto na razão de $100 \%$ da espécie do carrapato, quando comparada com sua confirmação por técnica de biologia molecular.

Os resultados das medições do idiossoma mostraram uma grande diferença entre as medidas de A. sculptum, que, no máximo, atingiram $0,498 \mathrm{~mm}$ e as medidas de $A$. dubitatum, que, no mínimo, registraram $0,554 \mathrm{~mm}$, conferindo baixo grau de incerteza à classificação dos espécimes, dentre as duas espécies de interesse.

É importante frisar que o presente trabalho se baseou em mensurações de espécimes que foram coletados em vida livre. Nesse caso, o comprimento do idiossoma é um caráter taxonômico altamente estável, pois todas as larvas originaram-se de ovos com tamanho típico para cada espécie (Barbieri et al., 2006). No caso de larvas já alimentadas, coletadas de hospedeiros, a mensuração do idiosoma perde seu caráter discriminatório entre as duas espécies de carrapatos, pois uma larva de A. sculptum em fase final de alimentação seria muito mais longa que uma larva de A. dubitatum em fase inicial de alimentação. Assim, as mensurações teriam que se basear em estruturas não expansivas do idiossoma, como, por exemplo, o escudo ou o gnatossoma.

Embora nenhuma outra espécie do gênero Amblyomma tenha sido testada neste estudo, tal técnica poderá ser utilizada nos locais onde se faz necessária uma vigilância acarológica, como proposta de identificação onde essas espécies prevalentes já são conhecidas, e a capivara é o principal hospedeiro, em áreas sob risco de transmissão de FMB, no estado de São Paulo e no sudeste do país. Oportunamente, é digno de nota que, em muitas áreas endêmicas para FMB associadas a capivaras no estado de São Paulo, $A$. sculptum e A. dubitatum têm sido as únicas espécies do gênero Amblyomma identificadas nos inquéritos acarológicos (Pinter et al., 2011). 


\section{CONCLUSÃO}

A aplicação dessa técnica de identificação morfométrica foi validada com significância estatística $\quad(\mathrm{P}<0,001), \quad$ sendo recomendada exclusivamente para diferenciação entre larvas das espécies A. dubitatum e A. sculptum (coletadas em vida livre) nos LPIs para FMB que apresentem uma ixodofauna já conhecida, constituída basicamente por apenas essas duas espécies de Amblyomma, tendo capivaras como hospedeiros primários.

\section{REFERÊNCIAS}

ALMEIDA, A.P.; CUNHA, L.M.; BELLO, A.C.P.P. et al. A novel Rickettsia infecting Amblyomma dubitatum ticks in Brazil. Ticks Tick Borne Dis., v.2, p.209-212, 2011.

BARBIERI, F.S.; BRITO, L.G.; LABRUNA, M.B. et al. Metodologia para o estudo da porotaxia em larvas de carrapatos Ixodidae. Porto Velho: EMBRAPA, 2006. p.1-5. (Comunicado Técnico n.321)

BEATI, L.; NAVA, S.; BURKMAN, E.J. et al. Amblyomma cajennense (Fabricius, 1787) (Acari: Ixodidae), the Cayenne tick: phylogeography and evidence for allopatric speciation. BMC Evol. Biol., v.13, p.267, 2013.

BLACK, W.C.; PIESMAN J. Phylogeny of hardand soft-tick taxa (Acari: Ixodida) based on mitochondrial 16S rDNA sequences. Proc. Natl. Acad. Sci. U.S.A., v.91, p.10034-10038, 1994.

BRITES-NETO, J.; NIERI-BASTOS, F.A.; BRASIL, J. et al. Environmental infestation and rickettsial infection in ticks in a Brazilian spotted fever-endemic area. Rev. Bras. Parasitol. Vet., v.22, p.367-372, 2013.

DANTAS-TORRES, F.; ONOFRIO, V.C.; BARROS-BATTESTI, D.M. The ticks (Acari: Ixodida: Argasidae, Ixodidae) of Brazil. Syst. Appl. Acarol., v.14, p.30-46, 2009.

ESTRADA-PEÑA, A.; TARRAGONA, E.L.; VESCO, U. et al. Divergent environmental preferences and areas of sympatry of tick species in the Amblyomma cajennense complex (Ixodidae). Int. J. Parasitol., v.44, p.1081-1089, 2014.

GUGLIELMONE， A.A.; ROBBINS， R.G.; APANASKEVICH, D.A. et al. The hard ticks of the world (Acari: Ixodida: Ixodidae). Dordrecht: Springer Science+Business Media, 2014. 738p.
KRAWCZAK, F.S.; MARTINS, T.F.; OLIVEIRA, C.S. et al. Amblyomma yucumense n. sp. (Acari: Ixodidae), a Parasite of wild mammals in Southern Brazil. J. Med. Entomol., v.52, p.28-37, 2015.

KRAWCZAK, F.S.; NIERI-BASTOS, F.A.; NUNES, F.P. et al. Rickettsial infection in Amblyomma cajennense ticks and capybaras (Hydrochoerus hydrochaeris) in a Brazilian spotted fever-endemic area. Parasite. Vectors, v.7, p.1-7, 2014.

LABRUNA, M.B. Brazilian spotted fever: the role of capybaras. In: MOREIRA, J.R.; FERRAZ, K.M.P.M.B.; HERRERA, E.A.; MACDONALD, D.W. (Eds.) Capybara: biology, use and conservation of an exceptional neotropical species. New York: Springer Science Business Media, 2013. chap.23, p.371-383.

LABRUNA, M.B.; PACHECO, R.C.; ATALIBA, A.C.; SZABÓ, M.P.J. Human Parasitism by the capybara tick Amblyomma dubitatum (Acari: Ixodidae). Entomol. News, v.118, p.77-80, 2007.

LABRUNA, M.B.; SANTOS, F.C.P.; OGRZEWALSKA, M. et al. Genetic identification of rickettsial isolates from fatal cases of Brazilian spotted fever and comparison with Rickettsia rickettsii isolates from the American Continents. J. Clin. Microbiol., v.52, p.3788-3791, 2014.

MANGOLD, A.J.; BARGUES, M.D.; MASCOMA, S. Mitochondrial 16S rDNA sequences and phylogenetic relationships of species of Rhipicephalus and other tick genera among Metastriata (Acari: Ixodidae). Parasitol. Res., v.84, p.478-484, 1998.

MARTINS, T.F.; BARBIERI, A.R.M.; COSTA, F.B. et al. Geographical distribution of Amblyomma cajennense (sensu lato) ticks (Parasitiformes: Ixodidae) in Brazil, with description of the nymph of A. cajennense (sensu stricto). Parasite. Vectors, v.9, p.186, 2016.

MARTINS, T.F.; ONOFRIO, V.C.; BARROSBATTESTI, D.M.; LABRUNA, M.B. Nymphs of the genus Amblyomma (Acari: Ixodidae) of Brazil: descriptions, redescriptions, and identification key. Ticks Tick Borne Dis., v.1, p.75-99, 2010. 
MATIAS, J.; GARCIA, M.V.; CUNHA, R.C. et al. Spotted fever group Rickettsia in Amblyomma dubitatum tick from the urban area of Campo Grande, Mato Grosso do Sul, Brazil. Ticks Tickborne Dis., v.6, p.107-110, 2015.

NAVA, S.; BEATI, L.; LABRUNA, M.B. et al. Reassessment of the taxonomic status of Amblyomma cajennense (Fabricius, 1787) with the description of three new species, Amblyomma tonelliae n. sp., Amblyomma interandinum n. sp. and Amblyomma patinoi n. sp., and reinstatement of Amblyomma mixtum Koch, 1844, and Amblyomma sculptum Berlese, 1888 (Ixodida: Ixodidae). Ticks Tick Borne Dis., v.5, p.252-276, 2014.

NAVA, S.; VENZAL, J.M.; LABRUNA, M.B. et al. Hosts, distribution and genetic divergence (16S rDNA) of Amblyomma dubitatum (Acari: Ixodidae). Exp. Appl. Acarol., v.51, p.335-351, 2010.

OGRZEWALSKA, M.; SARAIVA, D.G.; MORAES-FILHO, J. et al. Epidemiology of Brazilian spotted fever in the Atlantic Forest, state of São Paulo, Brazil. Parasitology, v.139, p.1283-1300, 2012.

ONOFRIO, V.C.; LABRUNA, M.B.; PINTER, A. et al. Comentários e chaves para as espécies do gênero Amblyomma. In: BARROSBATTESTI, D.M.; ARZUA, M.; BECHARA, G.H. Carrapatos de importância médicoveterinária da Região Neotropical: um guia ilustrado para identificação de espécies. São Paulo: Vox/ICTTD-3/Butantan, 2006. p.53-71.

PALLINI, A.; FADINI, M.A.M.; VENZON, M. et al. Demandas e perspectivas para a Acarologia no Brasil. Neotrop. Biol. Conserv., v.2, p.169175, 2007.

PAROLA, P.; PADDOCK, C.D.; SOCOLOVSCHI, C. et al. Update on tick-borne rickettsioses around the world: a geographic approach. Clin. Microbiol. Rev., v.26, p.657-702, 2013.

PFÄFFLE, M.; LITTWIN, N.; MUDERS, S.V.; PETNEY, T.N. The ecology of tick-borne diseases. Int. J. Parasitol., v.43, p.1059-1077, 2013.
PINTER, A.; COSTA, C.S.; HOLCMAN, M.M. et al. A Febre maculosa brasileira na região Metropolitana de São Paulo. Bol. Epidemiol. Paulista, v.13, p.1-45, 2016.

PINTER, A.; FRANÇA, A.C.; SOUZA, C.E. et al. Febre maculosa brasileira. Bol. Epidemiol. Paulista, Supl., v.8, p.1-31, 2011.

RAMOS, V.N.; OSAVA, C.F.; PIOVEZAN, U.; SZABÓ, M.P.J. Complementary data on four methods for sampling free-living ticks in the Brazilian Pantanal. Braz. J. Vet. Parasitol., v.23, p.516-521, 2014.

SAKAI, R.K.; COSTA, F.B.; UENO, T.E.H. et al. Experimental infection with Rickettsia rickettsii in an Amblyomma dubitatum tick colony, naturally infected by Rickettsia bellii. Ticks Tick Borne Dis., v.5, p.917-923, 2014.

SANGER, F.; NICKLEN, S.; COULSON, A.R. DNA sequencing with chain-terminating inhibitors. Proc. Nati. Acad. Sci. USA, v.74, p.5463-5467, 1977.

SONENSHINE, D.E.; ROE, M. Biology of ticks. 2.ed. New York: Oxford University Press, 2014. 2v., 496p.

SOUZA, C.E.; MORAES-FILHO, J.; OGRZEWALSKA, M. et al. Experimental infection of capybaras Hydrochoerus hydrochaeris by Rickettsia rickettsii and evaluation of the transmission of the infection to ticks Amblyomma cajennense. Vet. Parasitol., v.161, p.116-121, 2009.

SOUZA, S.S.A.L.; SOUZA, C.E.; NETO, E.J.R.; PRADO, A.P. Dinâmica sazonal de carrapatos (Acari: Ixodidae) na mata ciliar de uma área endêmica para febre maculosa na região de Campinas, São Paulo, Brasil. Cienc. Rural, v.36, p.887-891, 2006.

SZABÓ, M.P.J.; PINTER, A.; LABRUNA, M.B. Ecology, biology and distribution of spottedfever tick vectors in Brazil. Front. Cell. Infect. Microbiol., v.3, p.1-9, 2013.

WILSON, J.G.; KINZER, D.R.; SAUER, J.R.; HAIR, J.A. Chemo-attraction in the lone star tick (Acarina: Ixodidae). I. Response of different developmental stages to carbon dioxide administered via traps. J. Med. Entomol., v.9, p.245-252, 1972. 